International Electronic Journal of Geometry

Volume 8 No. 2 PP. 34-45 (2015) C IEJG

\title{
RICCI SOLITONS IN 3-DIMENSIONAL NORMAL ALMOST PARACONTACT METRIC MANIFOLDS
}

\author{
SELCEN YÜKSEL PERKTAŞ AND SADIK KELEŞ
}

(Communicated by Murat TOSUN)

\begin{abstract}
In the present paper we study 3-dimensional normal almost paracontact metric manifolds admitting Ricci solitons and gradient Ricci solitons. We give an example of 3-dimensional normal almost paracontact metric manifold. It is shown that if in a 3-dimensional normal almost paracontact metric manifold with $\alpha, \beta=$ constant the metric is Ricci soliton, where potential vector field $V$ is collinear with the characteristic vector field $\xi$, then the manifold is $\eta$-Einstein. We also prove that an $\eta$-Einstein 3-dimensional normal almost paracontact metric manifold with $\alpha, \beta=$ constant and $V=\xi$ admits a Ricci soliton. Furthermore, we show that if a 3-dimensional normal almost paracontact metric manifold admits a Ricci soliton $(g, \xi, \lambda)$ then the Ricci soliton is shrinking.
\end{abstract}

\section{INTRODUCTION}

The notion of Ricci soliton was introduced by Hamilton [17] in 1982. A Ricci soliton is a natural generalization of an Einstein metric. A pseudo-Riemannian manifold $(M, g)$ is called a Ricci soliton if it admits a smooth vector field $V$ (potential vector field) on $M$ such that

$$
\frac{1}{2}\left(£_{V} g\right)(X, Y)+S(X, Y)+\lambda g(X, Y)=0,
$$

where $£_{V}$ denotes the Lie-derivative in the direction $V, \lambda$ is a constant and $X, Y$ are arbitrary vector fields on $M$. A Ricci soliton is said to be shrinking, steady or expanding according to $\lambda$ being negative, zero or positive, respectively. It is obvious that a trivial Ricci soliton is an Einstein manifold with $V$ zero or Killing. Since Ricci solitons are the fixed points of the Ricci flow, they are important in understanding Hamilton's Ricci flow [18] : $\frac{\partial}{\partial t} g_{i j}=-2 S_{i j}$, viewed as a dynamical system, on the space of Riemannian metrics modulo diffeomorphisms and scalings.

Date: Received: September 9, 2013 and Accepted: March 12, 2014

2010 Mathematics Subject Classification. 53C15, 53C50.

Key words and phrases. Normal almost paracontact metric manifold, Ricci soliton, gradient Ricci soliton, $\eta$-Einstein manifold.

This article is the written version of author's plenary talk delivered on August 26-29, 2013 at 2nd International Euroasian Conference on Mathematical Sciences and Applications IECMSA2013 in Sarajevo, BOSNIA and HERZEGOVINA. 
In differential geometry, the Ricci flow is an intrinsic geometric flow. It is a process that deforms the metric of a Riemannian manifold in a way formally analogous to the diffusion of heat, smoothing out irregularities in the metric.

The vector field $V$ generates the Ricci soliton viewed as a special solution of the Ricci flow, and is called the generating vector field. A Ricci soliton is said to be a gradient Ricci soliton if the generating vector field $V$ is the gradient of a potential function $-f$, that is $V=-\nabla f$.

Some basic facts about Ricci solitons are given in following:

1. Compact steady or expanding solitons are Einstein in all dimensions [17, 19];

2. Compact shrinking solitons in dimension $n=2$ and $n=3$ must be of positive constant curvature $[18,19]$;

3. Compact Ricci solitons are always gradient Ricci solitons [6, 25].

If the manifold is Euclidean space, or more generally Ricci-flat, then Ricci flow leaves the metric unchanged. Conversely, any metric unchanged by Ricci flow is Ricci-flat. For a compact Einstein manifold, the metric is unchanged under normalized Ricci flow. Conversely, any metric unchanged by normalized Ricci flow is Einstein.

Geometric flows, especially Ricci flows, have become important tools in theoretical physics. Ricci soliton is known as quasi Einstein metric in physics literature and solutions of the Einstein field equations correspond to Ricci solitons [1, 14]. See also, [10, 22, 31].

Sharma [26] initiated the study of Ricci solitons in contact geometry as a $K$ contact metric. The authors in [16] studied gradient Ricci soliton of a non-Sasakian $(\kappa, \mu)$-contact manifold. Sharma and Ghosh [27] proved that Sasakian 3-manifold as a Ricci soliton represents the Heisenberg group. We also refer [11, 15, 23, 28, 29] for further read.

In spite of introducing and studying firstly in Riemannian geometry, the Ricci soliton equation has recently been investigated in pseudo-Riemannian context, especially in Lorentzian case (see $[4,5,24])$.

These circumstances motivated us to study the Ricci solitons in paracontact geometry. As a first step we consider 3-dimensional normal almost paracontact metric manifolds.

The study of paracontact geometry was initiated by Kaneyuki and Konzai in [20]. The authors defined almost paracontact structure on a pseudo-Riemannian manifold $M$ of dimension $(2 n+1)$ and constructed the almost paracomplex structure on $M^{2 n+1} \times \mathbb{R}$. Recently, Zamkovoy [34] studied paracontact metric manifolds and some important subclasses like para-Sasakian manifolds. Especially, in the recent years, many authors $[2,3,7,8,13]$ have pointed out the importance of paracontact geometry, and in particular of para-Sasakian geometry, by several papers giving the relationships with the theory of para-Kähler manifolds and its role in pseudoRiemannian geometry and mathematical physics.

The paper is organized as follows. Section 2 is devoted to some basic definitions for 3-dimensional normal almost paracontact metric manifolds. In section 3 , we obtain some curvature identities for a 3-dimensional normal almost paracontact metric manifold and construct an example. In section 4 , we prove that if a 3-dimensional non-paracosymplectic normal almost paracontact metric manifold with $\alpha, \beta=$ constant admits a Ricci soliton and $V$ is pointwise collinear with the 
structure vector field $\xi$, then $V$ is a constant multiple of $\xi$, the manifold is an $\eta$ Einstein manifold and the Ricci soliton is shrinking. Moreover, we show that if a 3-dimensional non-paracosymplectic normal almost paracontact metric manifold with $\alpha, \beta=$ constant is an $\eta$-Einstein manifold then the manifold admits a Ricci soliton. In section 5, we obtain some results for a 3-dimensional normal almost paracontact metric manifold admitting gradient Ricci soliton.

\section{3-DIMENSIONAL NORMAL ALMOST PARACONTACT METRIC MANIFOLDS}

A differentiable manifold $M$ of dimension $(2 n+1)$ is called almost paracontact manifold with the almost paracontact structure $(\varphi, \xi, \eta)$ if it admits a tensor field $\varphi$ of type $(1,1)$, a vector field $\xi$, a 1 -form $\eta$ satisfying the following conditions [20]:

$$
\begin{gathered}
\varphi^{2}=I-\eta \otimes \xi, \\
\eta(\xi)=1, \quad \varphi \xi=0,
\end{gathered}
$$

where $I$ denotes the identity transformation. Moreover the tensor field $\varphi$ induces an almost paracomplex structure on the paracontact distribution $D=\operatorname{ker} \eta$, i.e. the eigendistributions $D^{ \pm}$corresponding to the eigenvalues \pm 1 of $\varphi$ are both $n$ dimensional. As an immediate consequences of the conditions (2.1) and (2.2) we have

$$
\eta \circ \varphi=0, \quad \operatorname{rank}(\varphi)=2 n .
$$

If a $(2 n+1)$-dimensional almost paracontact manifold $M$ with an almost paracontact structure $(\varphi, \xi, \eta)$ admits a pseudo-Riemannian metric $g$ such that [34]

$$
g(\varphi X, \varphi Y)=-g(X, Y)+\eta(X) \eta(Y), \quad X, Y \in T M,
$$

then we say that $M$ is an almost paracontact metric manifold with an almost paracontact metric structure $(\varphi, \xi, \eta, g)$ and such metric $g$ is called compatible metric. Any compatible metric $g$ is necessarily of signature $(n+1, n)$.

From (2.3) it can be easily seen that [34]

$$
\begin{gathered}
g(X, \varphi Y)=-g(\varphi X, Y), \\
g(X, \xi)=\eta(X),
\end{gathered}
$$

for any $X, Y \in T M$. The fundamental 2 -form of $M$ is defined by

$$
\Phi(X, Y)=g(X, \varphi Y) .
$$

An almost paracontact metric structure becomes a paracontact metric structure if

$$
g(X, \varphi Y)=d \eta(X, Y),
$$

for all vector fields $X, Y$, where $d \eta(X, Y)=\frac{1}{2}\{X \eta(Y)-Y \eta(X)-\eta([X, Y])\}$.

For a $(2 n+1)$-dimensional manifold $M$ with an almost paracontact metric structure $(\varphi, \xi, \eta, g)$ one can also construct a local orthonormal basis. Let $U$ be coordinate neighborhood on $M$ and $X_{1}$ any unit vector field on $U$ orthogonal to $\xi$. Then $\varphi X_{1}$ is a vector field orthogonal to both $X_{1}$ and $\xi$, and $\left|\varphi X_{1}\right|^{2}=-1$. Now choose a unit vector field $X_{2}$ orthogonal to $\xi, X_{1}$ and $\varphi X_{1}$. Then $\varphi X_{2}$ is also vector field orthogonal to $\xi, X_{1}, \varphi X_{1}$ and $X_{2}$ and $\left|\varphi X_{2}\right|^{2}=-1$. Proceeding in this way we obtain a local orthonormal basis $\left(X_{i}, \varphi X_{i}, \xi\right),(i=1,2, \ldots, n)$, called a $\varphi$-basis [34].

An almost paracontact metric manifold is said to be normal if [21]

$$
N(X, Y)-2 d \eta(X, Y) \xi=0
$$


where $N$ is the Nijenhuis torsion tensor of $\varphi$ given by

$$
N(X, Y)=\varphi^{2}[X, Y]+[\varphi X, \varphi Y]-\varphi[\varphi X, Y]-\varphi[X, \varphi Y]
$$

for all $X, Y \in T M$. The normality condition says that the almost paracomplex structure $J$ on $M^{2 n+1} \times \mathbb{R}$ is defined by [21]

$$
J\left(X, f \frac{d}{d t}\right)=\left(\varphi X+f \xi, \eta(X) \frac{d}{d t}\right)
$$

is integrable (paracomplex).

The following Proposition presents conditions equivalent to the normality of 3dimensional almost paracontact metric manifold for later use.

Proposition 2.1. [32] For a 3-dimensional almost paracontact metric manifold $M$, the following three conditions are mutually equivalent

(i) $M$ is normal,

(ii) there exists functions $\alpha, \beta$ on $M$ such that

$$
\left(\nabla_{X} \varphi\right) Y=\beta(g(X, Y) \xi-\eta(Y) X)-\alpha(g(\varphi X, Y) \xi-\eta(Y) \varphi X),
$$

(iii) there exist functions $\alpha, \beta$ on $M$ such that

$$
\nabla_{X} \xi=\alpha(X-\eta(X) \xi)+\beta \varphi X .
$$

Corollary 2.1. [32] The functions $\alpha, \beta$ realizing (2.9) as well as (2.10) are given by

$$
2 \alpha=\operatorname{trace}\left\{X \rightarrow \nabla_{X} \xi\right\}, \quad 2 \beta=\operatorname{trace}\left\{X \rightarrow \varphi \nabla_{X} \xi\right\} .
$$

A 3-dimensional normal almost paracontact metric manifold is said to be

- paracosymplectic [9] if $\alpha=\beta=0$,

- quasi-para-Sasakian [12,32] if and only if $\alpha=0$ and $\beta \neq 0$,

- $\beta$-para-Sasakian [32,34] if and only if $\alpha=0, \beta \neq 0$ and $\beta$ is constant, in particular, para-Sasakian if $\beta=-1$,

- $\alpha$-para-Kenmotsu [33] if $\alpha \neq 0$ and $\alpha$ is constant and $\beta=0$.

\section{Some BASIC CURVATURE IDENTITIES}

In this section we obtain some curvature identities for a 3-dimensional normal almost paracontact metric manifold.

Let $M$ be a 3-dimensional normal almost paracontact metric manifold. Then we have

$$
\begin{gathered}
R(X, Y) \xi=-\left\{Y \alpha+\left(\alpha^{2}+\beta^{2}\right) \eta(Y)\right\} \varphi^{2} X+\left\{X \alpha+\left(\alpha^{2}+\beta^{2}\right) \eta(X)\right\} \varphi^{2} Y \\
1) \\
-\{Y \beta+2 \alpha \beta \eta(Y)\} \varphi X+\{X \beta+2 \alpha \beta \eta(X)\} \varphi Y, \\
\text { 2) } \\
S(Y, \xi)=-Y \alpha-\left(\xi \alpha+2\left(\alpha^{2}+\beta^{2}\right)\right) \eta(Y)+\varphi Y \beta \\
\xi \beta+2 \alpha \beta=0,
\end{gathered}
$$

for all $X, Y \in T M$.

In a 3 -dimensional semi-Riemannian manifold, it is well known that the curvature tensor always satisfies

$$
\begin{aligned}
R(X, Y) Z= & g(Y, Z) Q X-g(X, Z) Q Y+S(Y, Z) X-S(X, Z) Y \\
& -\frac{r}{2}(g(Y, Z) X-g(X, Z) Y)
\end{aligned}
$$


where $X, Y, Z \in T M, Q$ is the Ricci operator and $r$ is the scalar curvature of the manifold [30].

Now let $\widetilde{R}(X, Y, Z, W)=g(R(X, Y) Z, W)$, for all $X, Y, Z \in T M$. From (3.4) and (3.1) we have

$$
\widetilde{R}(\xi, Y, Z, \xi)=\left(\xi \alpha+\left(\alpha^{2}+\beta^{2}\right)\right) g(\varphi Y, \varphi Z) .
$$

Proposition 3.1. For a 3-dimensional normal almost paracontact metric manifold with $\alpha, \beta=$ constant, we have

$$
\begin{gathered}
S(X, Y)=-\left(\frac{r}{2}+\alpha^{2}+\beta^{2}\right) g(\varphi X, \varphi Y)+2\left(\alpha^{2}+\beta^{2}\right) \eta(X) \eta(Y), \\
Q X=\left(\frac{r}{2}+\alpha^{2}+\beta^{2}\right) X+\left(-\frac{r}{2}+\alpha^{2}+\beta^{2}\right) \eta(X) \xi,
\end{gathered}
$$

for all $X, Y \in T M$.

Proof. From (3.2), (3.4) and (3.5), the proof is straightforward.

Using (3.6) in (3.4), we have following

Lemma 3.1. Let $M$ be a 3-dimensional normal almost paracontact metric manifold with $\alpha, \beta=$ constant, then the Riemannian curvature tensor satisfies

$$
\begin{aligned}
R(X, Y) Z= & \left(\frac{r}{2}+2\left(\alpha^{2}+\beta^{2}\right)\right)(g(Y, Z) X-g(X, Z) Y) \\
& -g(X, Z)\left(-\frac{r}{2}+\alpha^{2}+\beta^{2}\right) \eta(Y) \xi \\
& +\left(-\frac{r}{2}+\alpha^{2}+\beta^{2}\right) \eta(Y) \eta(Z) X \\
& +g(Y, Z)\left(-\frac{r}{2}+\alpha^{2}+\beta^{2}\right) \eta(X) \xi \\
& -\left(-\frac{r}{2}+\alpha^{2}+\beta^{2}\right) \eta(X) \eta(Z) Y,
\end{aligned}
$$

where $X, Y, Z \in T M$ and $r$ is the scalar curvature.

From (3.3), we also have

Proposition 3.2. Let $M$ be a 3-dimensional normal almost paracontact metric manifold. If $\alpha, \beta=$ constant, then $M$ is either $\beta$-para-Sasakian, $\alpha$-para-Kenmotsu or paracosymplectic.

Note that $\beta$-para-Sasakian manifolds are quasi-para-Sasakian.

Now we shall give an example of 3-dimensional normal almost paracontact metric manifold.

Example 3.1. Let $M$ be the 3-dimensional real number space endowed with a coordinate system $(x, y, z)$ such that $z \neq 0$ and a semi-Riemannian metric of index 1 which is defined by

$$
g=\frac{d x^{2}-d y^{2}+d z^{2}}{z^{2}}
$$

We can define an orthonormal basis for the tangent space of $M$ by

$$
e_{1}=z \frac{\partial}{\partial x}, \quad e_{2}=z \frac{\partial}{\partial y}, \quad e_{3}=z \frac{\partial}{\partial z} .
$$

Now we consider

$$
\varphi e_{1}=e_{2}, \quad \varphi e_{2}=e_{1}, \quad \varphi e_{3}=0
$$




$$
\xi=e_{3}, \quad \eta(Z)=g\left(Z, e_{3}\right), \quad \text { for all } Z \in T M .
$$

Then we have

$$
\begin{gathered}
\eta\left(e_{3}\right)=1, \\
\varphi^{2} Z=Z-\eta(Z) \xi
\end{gathered}
$$

and

$$
g(\varphi Z, \varphi W)=-g(Z, W)+\eta(Z) \eta(W),
$$

for all $Z, W \in T M$, which imply that the set $(\varphi, \xi, \eta, g)$ is an almost paracontact metric structure on $M$.

The nonvanishing Christoffel symbols of $M$ are

$$
\Gamma_{13}^{1}=-\Gamma_{11}^{3}=\Gamma_{22}^{3}=\Gamma_{23}^{2}=\Gamma_{33}^{3}=-\frac{1}{z} .
$$

Then for the covariant derivatives of the Levi-Civita connection of the metric $g$ defined by (3.9), we have

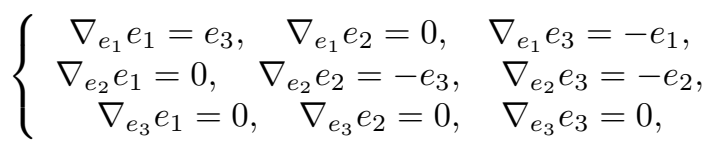

where $\left\{e_{1}, e_{2}, e_{3}\right\}$ is the orthonormal basis for the tangent space given by (3.10). From (3.11) we see that the equation (2.9) is satisfied for $\alpha=-1$ and $\beta=0$. Hence, $M$ is a 3 -dimensional normal almost paracontact metric manifold with $\alpha, \beta=$ constant.

\section{Ricci Soliton}

In this section, we consider a 3 -dimensional normal almost paracontact metric manifold $M$ admitting a Ricci soliton defined by (1.1). Let $V$ be a pointwise collinear vector field with the structure vector field $\xi$, that is $V=b \xi$, where $b$ is a function on $M$. From (1.1) we write

$$
g\left(\nabla_{X} b \xi, Y\right)+g\left(X, \nabla_{Y} b \xi\right)+2 S(X, Y)+2 \lambda g(X, Y)=0
$$

for $X, Y \in T M$. Then, we have

$$
\begin{gathered}
(X b) \eta(Y)+b g\left(\nabla_{X} \xi, Y\right)+(Y b) \eta(X) \\
+b g\left(X, \nabla_{Y} \xi\right)+2 S(X, Y)+2 \lambda g(X, Y)=0,
\end{gathered}
$$

which implies

$$
\begin{gathered}
(X b) \eta(Y)+(Y b) \eta(X)+2 \alpha b g(X, Y) \\
-2 \alpha b \eta(X) \eta(Y)+2 S(X, Y)+2 \lambda g(X, Y)=0,
\end{gathered}
$$

by virtue of (2.10). By putting $Y=\xi$ in (4.3) and using (3.6) we obtain

$$
X b+(\xi b) \eta(X)+4\left(\alpha^{2}+\beta^{2}\right) \eta(X)+2 \lambda \eta(X)=0 .
$$

Taking $X=\xi$ in the previous equation gives

$$
\xi b=-\left(2\left(\alpha^{2}+\beta^{2}\right)+\lambda\right) .
$$

If we replace (4.5) in (4.4), we get

$$
X b=-\left(2\left(\alpha^{2}+\beta^{2}\right)+\lambda\right) \eta(X),
$$

which yields

$$
d b=-\left(2\left(\alpha^{2}+\beta^{2}\right)+\lambda\right) \eta .
$$


Applying $d$ on both sides of the last equation, we have

$$
\left(2\left(\alpha^{2}+\beta^{2}\right)+\lambda\right) d \eta=0 .
$$

In a normal almost paracontact metric manifold, since $d \eta \neq 0$ we have

$$
2\left(\alpha^{2}+\beta^{2}\right)=-\lambda
$$

which implies

$$
d b=0 \text {, that is, } b=\text { constant, }
$$

by virtue of (4.6). Thus, using constancy of $b$ in (4.3) we obtain

$$
S(X, Y)=-(\lambda+\alpha b) g(X, Y)+\alpha b \eta(X) \eta(Y),
$$

for all $X, Y \in T M$. Hence we have,

Theorem 4.1. Let $M$ be a 3-dimensional non-paracosymplectic normal almost paracontact metric manifold with $\alpha, \beta=$ constant. If $M$ admits a Ricci soliton and $V$ is pointwise collinear with the structure vector field $\xi$, then $V$ is a constant multiple of $\xi$ and $M$ is an $\eta$-Einstein manifold.

Let assume the converse, that is, let $M$ be a 3-dimensional $\eta$-Einstein normal almost paracontact metric manifold with $\alpha, \beta=$ constant and $V=\xi$. Then we can write

$$
S(X, Y)=\mu g(X, Y)+\rho \eta(X) \eta(Y),
$$

where $\mu, \rho$ are scalars and $X, Y \in T M$. From (2.10) we have

$$
\begin{aligned}
\left(£_{\xi} g\right)(X, Y) & =g\left(\nabla_{X} \xi, Y\right)+g\left(X, \nabla_{Y} \xi\right) \\
& =2 \alpha g(X, Y)-2 \alpha \eta(X) \eta(Y),
\end{aligned}
$$

which implies that

$$
\begin{aligned}
\left(£_{\xi} g\right)(X, Y)+2 S(X, Y)+2 \lambda g(X, Y)= & 2(\alpha+\mu+\lambda) g(X, Y) \\
& -2(\alpha-\rho) \eta(X) \eta(Y) .
\end{aligned}
$$

From the previous equation it is obvious that $M$ admits a Ricci soliton $(g, \xi, \lambda)$ if

$$
\alpha+\mu+\lambda=0
$$

and

$$
\rho=\alpha=\text { constant. }
$$

Equating the right hand sides of (3.6) and (4.9) and taking $X=Y=\xi$ gives

$$
2\left(\alpha^{2}+\beta^{2}\right)=\mu+\rho,
$$

that is,

$$
\mu=2\left(\alpha^{2}+\beta^{2}\right)-\alpha=\text { constant }
$$

Thus, we get

Theorem 4.2. Let $M$ be a 3-dimensional non-paracosymplectic normal almost paracontact metric manifold with $\alpha, \beta=$ constant. If $M$ is an $\eta$-Einstein manifold with $S=\mu g+\rho \eta \otimes \eta$, then the manifold admits a Ricci soliton $(g, \xi,-(\mu+\rho))$. 
Now taking $V=\xi$, the equation (1.1) becomes

$$
\left(£_{\xi} g\right)(X, Y)+2 S(X, Y)+2 \lambda g(X, Y)=0,
$$

for all $X, Y \in T M$. By using (2.10) it is easy to see that

$$
\left(£_{\xi} g\right)(X, Y)=2 \alpha(g(X, Y)-\eta(X) \eta(Y)) .
$$

Using (4.13) and (3.6) we have

$$
\begin{aligned}
\left(£_{\xi} g\right)(X, Y)+2 S(X, Y)= & \left\{r+2\left(\alpha^{2}+\beta^{2}\right)\right\} g(X, Y) \\
& -\left\{r-2\left(\alpha^{2}+\beta^{2}-\alpha\right)\right\} \eta(X) \eta(Y) .
\end{aligned}
$$

Replacing the last equation in (4.12) we obtain

(4.15) $\left\{r+2\left(\alpha^{2}+\beta^{2}+\alpha+\lambda\right)\right\} g(X, Y)-\left\{r-2\left(\alpha^{2}+\beta^{2}-\alpha\right)\right\} \eta(X) \eta(Y)=0$.

Putting $X=Y=\xi$ in (4.15) gives

$$
\lambda=-\left(\alpha^{2}+\beta^{2}\right) .
$$

Thus, we have

Theorem 4.3. If a 3-dimensional non-paracosymplectic normal almost paracontact metric manifold with $\alpha, \beta=$ constant admits a Ricci soliton $(g, \xi, \lambda)$ then the Ricci soliton is shrinking.

Now let us denote $£_{\xi} g+2 S$ by $B$. Then from (4.14) we write (4.16) $B(X, Y)=\left\{r+2\left(\alpha^{2}+\beta^{2}\right)\right\} g(X, Y)-\left\{r-2\left(\alpha^{2}+\beta^{2}-\alpha\right)\right\} \eta(X) \eta(Y)$, for any $X, Y \in T M$. Taking into account (4.12) it is obvious that $B$ is a parallel symmetric $(0,2)$-tensor field. Thus using (4.16) we have

$$
\begin{aligned}
0= & \left(\nabla_{U} B\right)(X, Y) \\
= & d r(U)\{g(X, Y)-\eta(X) \eta(Y)\} \\
& -\left\{r-2\left(\alpha^{2}+\beta^{2}-\alpha\right)\right\}\left\{\left(\nabla_{U} \eta\right)(X) \eta(Y)+\eta(X)\left(\nabla_{U} \eta\right)(Y)\right\} .
\end{aligned}
$$

Taking $X=Y=e_{i}$ in the last equation above we get

$$
d r=0 .
$$

Here $\left\{e_{i}\right\}, i=1,2,3$, is an orthonormal basis of $T_{p} M, \forall p \in M$. So we have

Theorem 4.4. If a 3-dimensional non-paracosymplectic normal almost paracontact metric manifold $M$ with $\alpha, \beta=$ constant admits a Ricci soliton $(g, \xi, \lambda)$ then $M$ is of constant scalar curvature.

\section{Gradient Ricci Soliton}

A Ricci soliton is said to be a gradient Ricci soliton if the generating vector field $V$ is the gradient of a potential function $-f$, that is $V=-\nabla f$. In this case (1.1) takes the form

$$
\nabla \nabla f=S+\lambda g
$$

Let $M$ be a 3 -dimensional non-paracosymplectic normal almost paracontact metric manifold with $\alpha, \beta=$ constant. From (5.1) for any $Y \in T M$ we write

$$
\nabla_{Y} \operatorname{gradf}=Q Y+\lambda Y \text {, }
$$

which gives

$$
R(X, Y) \operatorname{gradf}=\left(\nabla_{X} Q\right) Y-\left(\nabla_{Y} Q\right) X
$$


where $Q$ is the Ricci operator of the manifold and $X \in T M$. By using (3.7) we get

$$
\begin{aligned}
\left(\nabla_{U} Q\right) X= & \frac{d r(U)}{2}(X-\eta(X) \xi) \\
& +\left(-\frac{r}{2}+\alpha^{2}+\beta^{2}\right)\{\alpha[(g(X, U)-\eta(X) \eta(U)) \xi+U] \\
& +\beta[g(X, \varphi U) \xi+\eta(X) \varphi U]\},
\end{aligned}
$$

for all $U \in T M$. Taking $U=\xi$ in (5.4) we obtain

$$
\left(\nabla_{\xi} Q\right) X=\frac{d r(\xi)}{2}(X-\eta(X) \xi)-\left(-\frac{r}{2}+\alpha^{2}+\beta^{2}\right) \alpha(\eta(X)-1) \xi .
$$

If we put $X=\xi$ in (5.4) we also get

$$
\left(\nabla_{U} Q\right) \xi=-\left(-\frac{r}{2}+\alpha^{2}+\beta^{2}\right)(\alpha(\eta(U)-1) \xi-\beta \varphi U) .
$$

So from (5.5) and (5.6) we have

$$
g\left(\left(\nabla_{\xi} Q\right) X-\left(\nabla_{X} Q\right) \xi, \xi\right)=0,
$$

which implies

$$
g(R(\xi, X) \operatorname{grad} f, \xi)=0 .
$$

Using (3.5) in the last equation we obtain

$$
0=\left(\alpha^{2}+\beta^{2}\right)\{-g(X, \operatorname{grad} f)+\eta(X) \eta(\operatorname{grad} f)\} .
$$

Since $\alpha^{2}+\beta^{2} \neq 0$, we get

$$
g(X, \operatorname{grad} f)=\eta(X) \eta(\operatorname{grad} f)
$$

which implies

$$
\operatorname{grad} f=\xi(f) \xi
$$

Now using (5.11) in (5.1) we write

$$
\begin{aligned}
S(X, Y)+\lambda g(X, Y) & =g\left(\nabla_{Y} \operatorname{grad} f, X\right) \\
& =g\left(Y(\xi f) \xi+(\xi f) \nabla_{Y} \xi, X\right) .
\end{aligned}
$$

Thus, we have

$$
\begin{aligned}
S(X, Y)+\lambda g(X, Y)= & Y(\xi f) \eta(X) \\
& +(\xi f)\{\alpha(g(X, Y)-\eta(X) \eta(Y))+\beta g(X, \varphi Y)\},
\end{aligned}
$$

by virtue of (2.10). Taking $X=\xi$ in the last equation and using (3.6) we have

$$
Y(\xi f)=\left(-2\left(\alpha^{2}+\beta^{2}\right)+\lambda\right) \eta(Y) .
$$

Interchanging the roles of $X$ and $Y$ in (5.12) gives

$$
\begin{aligned}
S(Y, X)+\lambda g(Y, X)= & X(\xi f) \eta(Y) \\
& +(\xi f)\{\alpha(g(Y, X)-\eta(Y) \eta(X))+\beta g(Y, \varphi X)\} .
\end{aligned}
$$

Since the Ricci tensor and the metric is symmetric from (2.4), (3.1) and (3.3) we obtain

$$
\begin{aligned}
2 S(X, Y)+2 \lambda g(X, Y)= & X(\xi f) \eta(Y)+Y(\xi f) \eta(X) \\
& +2 \alpha(\xi f)(g(X, Y)-\eta(X) \eta(Y)),
\end{aligned}
$$


which implies

$$
\begin{aligned}
g(Q X, Y)+\lambda g(X, Y)= & \alpha(\xi f) g(X, Y) \\
& +\left(-2\left(\alpha^{2}+\beta^{2}\right)+\lambda-\alpha(\xi f)\right) \eta(X) \eta(Y)
\end{aligned}
$$

by virtue of (5.13). Then from (5.2) and (5.16) we get

$$
\nabla_{Y} \operatorname{grad} f=\alpha(\xi f) Y+\left(\lambda-2\left(\alpha^{2}+\beta^{2}\right)-\alpha(\xi f)\right) \eta(Y) \xi
$$

By using the last equation one obtains

$$
\begin{aligned}
R(X, Y) g r a d f= & \alpha\{X(\xi f) Y-Y(\xi f) X-(X(\xi f) \eta(Y)-Y(\xi f) \eta(X)) \xi\} \\
& +\left(-2\left(\alpha^{2}+\beta^{2}\right)+\lambda-\alpha(\xi f)\right)\left\{\left(\left(\nabla_{X} \eta\right) Y-\left(\nabla_{Y} \eta\right) X\right) \xi\right. \\
& \left.+\eta(Y) \nabla_{X} \xi-\eta(X) \nabla_{Y} \xi\right\}
\end{aligned}
$$

and from (5.11) we get

$$
0=g(R(X, Y) \operatorname{grad} f, \xi)=2 \beta\left(-2\left(\alpha^{2}+\beta^{2}\right)+\lambda-\alpha(\xi f)\right) g(\varphi X, Y),
$$

for all $X, Y \in T M$. Hence, we conclude

$$
\beta\left(-2\left(\alpha^{2}+\beta^{2}\right)+\lambda-\alpha(\xi f)\right)=0,
$$

which implies following three cases:

Case $I$ : If $\beta=0$ then the manifold is a $\alpha$-para-Kenmotsu manifold.

Case II : If $-2\left(\alpha^{2}+\beta^{2}\right)+\lambda-\alpha(\xi f)=0$, then from (5.13) we have

$$
Y(\xi f)=-\alpha(\xi f) \eta(Y) .
$$

Using the last expression, equation (5.13) reduces to

$$
S(X, Y)+\lambda g(X, Y)=\alpha(\xi f) g(X, Y) .
$$

Putting $X=Y=e_{i}$, where $\left\{e_{i}\right\}, i=1,2,3$, is an orthonormal basis of $T_{p} M$, $\forall p \in M$ in (5.22) and then taking a sum gives

$$
r+3 \lambda=3 \alpha(\xi f)
$$

which implies that $r=-3 \lambda$, that is, $r$ is a constant.

Case III : If $\beta=0$ and $-2\left(\alpha^{2}+\beta^{2}\right)+\lambda-\alpha(\xi f)=0$ then we get

$$
(\xi f)=-2 \alpha+\frac{\lambda}{\alpha}
$$

which implies that $(\xi f)=c=$ constant. Then from (5.11) we have

$$
d f(X)=c \eta(X) .
$$

Applying $d$ to the both sides of the last equation we get

$$
c d \eta=0 .
$$

Since $d \eta \neq 0$ then we see that $c=0$ and so $f$ is a constant function. Hence, (5.1) reduces to

$$
S=-\lambda g=-2 \alpha^{2} g .
$$

According to three cases given above, we have

Theorem 5.1. If a 3-dimensional non-paracosymplectic normal almost paracontact metric manifold with $\alpha, \beta=$ constant admits a gradient Ricci soliton then the manifold is an $\alpha$-para-Kenmotsu manifold, or of constant scalar curvature or Einstein. 


\section{REFERENCES}

[1] Akbar, M.M., Woolgar, E., Ricci solitons and Einstein-scalar field theory , Class. Quantum Grav., 26, 055015 (14pp), 2009, doi:10.1088/0264-9381/26/5/055015.

[2] Alekseevski, D. V., Cortés, V., Galaev, A. S., Leistner, T. , Cones over pseudo-Riemannian manifolds and their holonomy, J. Reine Angew. Math., 635 (2009), 23-69.

[3] Alekseevski, D. V., Medori, C., Tomassini, A., Maximally homogeneous para-CR manifolds, Ann. Global Anal. Geom., 30 (2006), 1-27.

[4] Brozos-Vazquez, M., Calvaruso, G., Garcia-Rio, E., Gavino-Fernandez, S., Three-dimensional Lorentzian Homogenous Ricci Solitons, Israel J Math 188 (2012), 385-403.

[5] Case, J. S., Singularity theorems and the Lorentzian splitting theorem for the Bakry Emery Ricci Tensor, Journal of Geometry and Physics 60 (2010), 477-490.

[6] Chow, B., Knopf, D., The Ricci flow: an introduction, volume 110 of Mathematical Surveys and Monographs, American Mathematical Society, Providence, RI, 2004.

[7] Cortés, V., Mayer, C., Mohaupt, T., Saueressing, F., Special geometry of Euclidean supersymmetry 1. Vector multiplets, J. High Energy Phys., 0403 (2004), 028, 73 pp.

[8] Cortés, V., Lawn, M. A., Schäfer, L., Affine hyperspheres associated to special para-Kähler manifolds, Int. J. Geom. Methods Mod. Phys., 3 (2006), 995-1009.

[9] Dacko, P., On almost paracosymplectic manifolds, Tsukuba J. Math. 28 (2004), no.1, 193-213.

[10] Das, S., Prabhu, K., Sayan K., Int. J. Geom. Methods Mod. Phys. 07, 837 (2010). DOI: $10.1142 / \mathrm{S} 0219887810004579$.

[11] De, U.C., Turan, M., Yıldız, A., De, A., Ricci solitons and gradient Ricci solitons on 3dimensional normal almost contact metric manifolds, Publ. Math. Debrecen, Ref. no.: 4947, (2012), 1-16.

[12] Erdem, S., On almost (para)contact (hyperbolic) metric manifolds and harmonicity of $\left(\varphi, \varphi^{\prime}\right)$ holomorphic maps between them, Houston J. Math., 28 (2002), 21-45.

[13] Erdem, S., On almost (para)contact (hyperbolic) metric manifolds and harmonicity of $\left(\varphi, \varphi^{\prime}\right)$ holomorphic maps between them, Houston J. Math., 28 (2002), 21-45.

[14] Friedan, D., Nonlinear models in $2+\varepsilon$ dimensions, Ann. Phys., 163(2), 318-419, 1985.

[15] Ghosh, A., Kenmotsu 3-metric as a Ricci soliton, Chaos, Solitons \& Fractals 44 (8), 2011, 647-650.

[16] Ghosh, A., Sharma, R., Cho, J.T., Contact metric manifolds with $\eta$-parallel torsion tensor, Annals of Global Analysis and Geometry, 34, 287-299, 2008.

[17] Hamilton, R. S., Three-manifolds with positive Ricci curvature. J. Di . Geo., 17:255-306,1982

[18] Hamilton, R. S., The Ricci flow on surfaces, Mathematics and general relativity (SantaCruz, CA,1986), Contemp. Math. 71, A.M.S., 237-262, 1988.

[19] Ivey, T., Ricci solitons on compact 3-manifolds, Differential Geo. Appl. 3, 301-307, 1993.

[20] Kaneyuki, S., Konzai, M., Paracomplex structure and affine symmetric spaces, Tokyo J. Math., 8 (1985), 301-308.

[21] Kaneyuki, S., Willams, F. L., Almost paracontact and parahodge structure on manifolds, Nagoya Math. J., 99, 173-187, 1985.

[22] Kholodenko, A. L., Towards physically motivated proofs of the Poincaré and the geometrization conjectures, Journal of Geometry and Physics 58, 259-290, 2008.

[23] Nagaraja, H.G., Premalatha, C.R., Ricci solitons in Kenmotsu manifolds, Journal of Mathematical Analysis, vol. 3, no. 2, pp. 18-24, 2012.

[24] Payne, T. L., The existence of soliton metrics for nilponent Lie Groups, Geometriae Dedicate 145 (2010), 71-88.

[25] Perelman, G., The entropy formula for the Ricci flow and its geometric applications, ArXiv:math.DG/0211159

[26] Sharma, R., Certain Results on $K$-Contact and $(k, \mu)$-Contact Manifolds, J. Geom. 89 (2008), 138-147.

[27] Sharma, R., Ghosh, A., Sasakian 3-manifolds as a Ricci soliton represents the Heisenberg group, International Journal of Geometric Methods in Modern Physics, 2011 08:01, 149-154

[28] Tripathi, M.M., Ricci solitons in contact metric manifolds, arXiv:0801.4222, 2008.

[29] Turan, M., De, U.C., Yıldız, A.,Ricci solitons and gradient Ricci solitons in threedimensional trans-Sasakian manifolds, Filomat, Volume 26, Issue 2, Pages: 363-370, 2012, doi:10.2298/FIL1202363T.

[30] Willmore, T.J., Differential Geometry, Clarendon Press, Oxford, 1958. 
[31] Woolgar, E., Some applications of Ricci flow in physics, Canadian Journal of Physics, 2008, 86(4): 645-651, 10.1139/p07-146.

[32] Welyczko, J., Legendre curves in 3-dimensional Normal almost paracontact metric manifolds, Result. Mth. 54 (2009), 377-387.

[33] Welyczko, J., Slant curves in 3-dimensional normal almost paracontact metric manifolds, Mediterr. J. Math. 11 (2014), no. 3, 965978.

[34] Zamkovoy, S.,Canonical connection on paracontact manifolds, Ann. Glob. Anal. Geo., 36 (2009), 37-60.

Adiyaman University, Faculty of Arts and Sciences, Department of Mathematics, 02040, Adiyaman/TURKEY.

E-mail address: sperktas@adiyaman.edu.tr

İNönü University, Faculty of Arts and Sciences, Department of Mathematics, 44280, MALATYA/TURKEY.

E-mail address: sadik.keles@inonu.edu.tr 